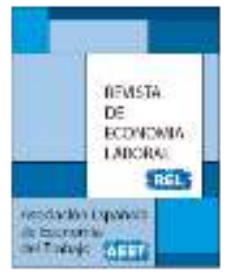

\title{
RECENSIÓN
}

Miguel Ángel Malo (Universidad de Salamanca) [Director]

Begoña Cueto (Universidad de Oviedo)

Ángel Luis Martín (Universidad de Valladolid)

Alfonso Moral (Universidad de Valladolid)

Almudena Moreno (Universidad de Valladolid)

\section{Empleo y formación a lo largo de la vida en Castilla y León}

\section{(Consejo Económico y Social de Castilla y León) Informe a iniciativa propia}

La formación a lo largo de la vida o aprendizaje permanente (lifelong learning en la literatura anglosajona) puede entenderse de dos maneras. Desde un punto de vista restrictivo, se define como educación para aquellos que ya han dejado el sistema educativo reglado en cualquier de sus niveles (con independencia de si trabajan o no). En una formulación más amplia, la formación a lo largo de la vida cubre precisamente toda la vida, atendiendo a todas las experiencias educativas y formativas de las personas antes, durante y después de su paso por el sistema educativo reglado. En este informe adoptaremos una perspectiva más próxima al punto de vista restrictivo, pero teniendo en cuenta que el diseño de cualquier estrategia de formación a lo largo de la vida tiene que partir necesariamente de los resultados del sistema reglado. La razón es que la mayor parte de las personas no vuelven a alterar su nivel de estudios tras salir del sistema reglado, por lo que se constituye en la base a partir de la cual se construyen las futuras actividades formativas y está directamente conectado con las posibilidades de empleo de las personas a lo largo de sus vidas.

Este tipo de aprendizaje permite a los trabajadores afrontar con mayores posibilidades de éxito momentos críticos de sus carreras

(C) Revista de Economía Laboral 
laborales como la transición del sistema educativo al mercado de trabajo o las transiciones entre empleos, incluso en contextos de elevado desempleo como el que actualmente se vive en países como España. En definitiva, la formación a lo largo de la vida deviene un elemento crucial para una mejor gestión de las carreras laborales y un instrumento de intervención de la Administración Pública para mejorar el bienestar de las personas a largo plazo. Adoptar la perspectiva del transcurso de la vida facilita la integración de diferentes tipos de intervenciones formativas que, de forma aislada, corren el riesgo de sobreproteger ciertos colectivos o de dejar otros sin una protección adecuada simplemente por no tener en cuenta que determinados problemas se acumulan más en unas carreras laborales que en otras.

Este informe pretende buscar una serie de elementos básicos para colaborar en la construcción de una estrategia de formación a lo largo de la vida en el ámbito de Castilla y León. No obstante, el enfoque del informe es sobre todo general, intentando alumbrar las cuestiones de largo plazo aunque en ocasiones estén más allá del ámbito de decisión estrictamente autonómico. El informe consta de dos secciones.

La sección 1 es una aproximación general hacia la evolución de la tasa de empleo a lo largo de la vida para diferentes niveles educativos en Castilla y León, distinguiendo los resultados por género. La sección 2 se centra en los datos sobre formación para el empleo en Castilla y León. Cuando lo permiten los datos disponibles se analiza la experiencia de Castilla y León en diferentes ámbitos formativos, básicamente tras el paso por el sistema educativo reglado comparando la experiencia autonómica con la del conjunto de España. Como aportación genuina de esta parte, cabe destacar que se han construido las series temporales más largas que permiten los datos publicados sobre formación a partir de diferentes fuentes. La finalidad de tener la visión de largo plazo que requiere un análisis sobre formación a lo largo de la vida.

\section{La evolución del empleo a lo largo de la vida}

A largo plazo, el mercado de trabajo de Castilla y León se caracteriza por un descenso tendencial de la tasa de actividad y una evolución de la tasa de empleo que no sube tanto en las expansiones como en el conjunto de España, al tiempo que la brecha de la tasa de paro por género no ha llegado a cerrarse como en España. Quizá esto se deba a la base demográfica de su mercado de trabajo, con un menor porcentaje de personas en las edades centrales de la vida, y relativamente mayor con certificado de escolaridad y formación profesional, aunque menos con 
Empleo y formación a lo largo de la vida en Castilla y León

estudios superiores.

En el conjunto de España, las tasas de empleo a lo largo de la vida muestran la concentración de empleo en las edades centrales para todas las cohortes, con un impacto negativo de la actual recesión en las generaciones que estaban en el momento crítico de su integración laboral al iniciarse la crisis y un gran bloqueo de la entrada al empleo de las generaciones jóvenes que han entrado durante la crisis. Castilla y León sigue la pauta general de España aunque el descenso en las tasas de empleo de las generaciones que tenían de 33 a 42 años en 2008 ha sido algo menor. Este impacto algo menor de la crisis se aprecia en todos los niveles de estudio, pero en especial en los que tienen estudios medios y universitarios.

Durante la actual recesión ha disminuido sensiblemente el tamaño de las cohortes de los nacidos en 1981-85 en general y los nacidos en 1976-80 entre los universitarios. Esto puede deberse a la aparente menor caída de la tasa de empleo de estas generaciones en Castilla y León respecto del conjunto de España. También se observa que las cohortes más jóvenes permanecen más tiempo enroladas en el sistema educativo. Este proceso es más acentuado en Castilla y León, especialmente en el caso de las mujeres. Finalmente, la inserción de los titulados universitarios de Castilla y León durante la crisis es significativa y con una pauta temporal semejante a la del conjunto de España, aunque es algo más baja en Castilla y León, sobre todo en el primer año tras finalizar los estudios.

A nivel nacional se observa que los adultos ocupados obtienen mejor rendimiento en el uso de competencias básicas (comprensión lectora y matemáticas) que los desempleados y que los inactivos, si bien los ocupados que están infracualificados o sobrecualificados en su puesto de trabajo tienen rendimientos más bajos de lo esperado en las competencias básicas (especialmente los sobrecualificados).

\section{La formación a lo largo de la vida}

La formación profesional reglada en Castilla y León tiene más peso sobre el conjunto de enseñanzas generales que en el conjunto de España, aumentando en las crisis y disminuyendo en las expansiones. Sin embargo, la evolución de la formación profesional ocupacional ha estado marcada por los planes FIP, con un descenso tendencial en el número medio de alumnos por curso y en la ratio de alumnos formados respecto de parados que es más intenso en Castilla y León. Por su parte, los alumnos de formación continua han crecido de forma sostenida, 
especialmente tras los dos cambios institucionales de 1994 y 2004 y su evolución ha sido más coincidente con España que en el caso de la formación profesional ocupacional. El peso de la formación continua en el total español se ha mantenido relativamente estable, con un ratio de participantes respecto de ocupados que también evoluciona de forma semejante en Castilla y León y en España, aunque presenta una ruptura en 2004 hacia un crecimiento más rápido que se mantiene durante la actual recesión.

También parece consolidarse el crecimiento de la oferta parcial de formación profesional, pieza clave de la flexibilidad en el seguimiento de la formación profesional de forma compatible con el trabajo o con otras actividades. Por familias profesionales, la formación para el empleo de demanda muestra que la composición y el peso relativo del alumnado son muy similares en Castilla y León y España. Dos familias profesionales crecieron en alumnado en los años previos a la crisis de empleo de 2008, "Administración y gestión" y "Seguridad y medio ambiente", alcanzando niveles significativamente superiores al resto.

Desde un punto de vista cualitativo, se constata la especial importancia de los certificados de profesionalidad de nivel 1 frente a las de nivel 2 y 3 , se aprecia también que es importante crear un sistema de incentivos en las empresas para que los certificados de profesionalidad cumplan su objetivo. A este respecto, las experiencias europeas resaltan el papel central de la validación de conocimientos profesionales en la formación permanente, aunque también ponen de manifiesto los desafíos que deben superarse y la legitimidad del proceso de certificación.

La cuantía de las subvenciones para la formación de demanda y de oferta (para ocupados y parados) ha descendido con claridad durante la crisis y, aunque en 2013, parecen haber repuntado (excepto la formación de oferta para desempleados) sus niveles son sustancialmente inferiores a los previos a la crisis. El amplio seguimiento en Castilla y León de formación no reglada de idiomas e informática es un indicador de la necesidad de dar prioridad a estos dos campos en cualquier estrategia regional de formación a lo largo de la vida, pero también muestra la necesidad de conseguir un dominio activo (sobre todo de las lenguas extranjeras) durante el paso por el sistema educativo reglado

Las iniciativas más recientes de la Unión Europea en el ámbito de la formación permanente están centradas en la educación reglada y, por tanto, en la primera fase de la vida, quedando el resto de etapas relativamente relegadas precisamente por el diseño del programa Erasmus+ (las movilidades Erasmus han crecido de forma generalizada entre 1994 a 2011). Los diferentes análisis muestran múltiples efectos beneficiosos sobre la inserción laboral de los beneficiarios del programa 
Erasmus, lo cual resalta por oposición la relevancia de los menores recursos que se dedican a este programa como resultado de las políticas de ajuste presupuestario. Los proyectos autonómicos dentro de Erasmus + muestran la relevancia del programa Comenius y se centran sobre todo en el fomento del aprendizaje de lenguas extranjeras y el desarrollo de contenidos basados en las nuevas tecnologías de la información y la comunicación, aunque también cabe resaltar las iniciativas dirigidas a la educación de adultos.

En el ámbito de la OCDE, desde 1996 las estrategias nacionales de formación a lo largo de la vida han generado una mejor estructuración de las cualificaciones, mayor flexibilidad del sistema educativo y mejor atención a la diversidad. Sin embargo, la formación a lo largo de la vida carece de una estructuración institucional adecuada, sirve mejor a quienes han tenido previamente éxito en el sistema reglado y sigue habiendo un bajo enrolamiento en actividades de formación por encima de los 30 años. Las buenas prácticas a nivel internacional buscan mejorar la situación de los jóvenes que abandonaron tempranamente sus estudios, la transformación de la educación de adultos y el reconocimiento de los aprendizajes realizados fuera del sistema reglado de formación. De forma transversal, el desarrollo de las cualificaciones de nivel 5 del Marco Europeo de Cualificaciones supone una oportunidad para desarrollar nuevas perspectivas del aprendizaje a lo largo de la vida, sobre todo ante la transformación de los puestos de trabajo por las nuevas tecnologías.

Castilla y León presenta mejores resultados relativos que el resto de comunidades autónomas en el nivel de competencias educativas de los jóvenes de 15 años (según el estudio PISA), si bien una parte de esa diferencia no se debería directamente al sistema educativo sino a una composición diferente del alumnado. La variedad autonómica muestra la existencia de dos sistemas, un modelo "propio" y un modelo "general", en los cuales los centros integrados tienen diferente grado de desarrollo y centralidad en el modelo, siendo menor en el "general" (posiblemente el más aplicable al caso de Castilla y León). Los datos de la Fundación Tripartita sobre formación profesional fuera del sistema reglado también muestran diferencias autonómicas relevantes en las tasas de cobertura de los diferentes tipos de formación no reglada, alcanzando Castilla y León elevadas tasas de cobertura en términos relativos salvo en el caso de la formación de demanda en términos de trabajadores cubiertos. 